\title{
Reversed Field Pinch Approach to Magnetic Fusion
}

\author{
S. Ortolani, Padova
}

(Istituto Gas lonizzati del C.N.R. Associazione Euratom-CNR)

The process whereby a current channel contracts under the influence of the magnetic field generated by the current itself, is known as the "pinch effect" and it was first studied by Bennett in 1934. It was also the first to be used experimentally (by Cousins and Ware in 1951) to confine plasma in a magnetic field in toroidal geometry. Indeed, during the 1950 s pinches, both linear and toroidal, were the most widely studied systems in fusion research.

The main observation from these early experiments was that the plasma was macroscopically unstable because of the fast growing magnetohydrodynamic (MHD) instabilities which destroyed the symmetry of the plasma column and led to wall contact.

The addition of a longitudinal magnetic field $\left(B_{z}\right.$ in cylindrical geometry, Fig. $1 ; B_{\phi}$ in toroidal geometry, Fig. 2) was suggested by theoretical studies and led to experiments on the so called "stabilized pinch" configuration (with $B_{\phi} \cong B_{\Theta}$ ). Although the macroscopic stability of the plasma improved, residual instabilities and fluctuations caused poor plasma confinement.

One possible way of overcoming the pinch instabilities, proposed during the 1960 s was the tokamak configuration in which a large toroidal field is applied so that $B_{\phi} / B_{\Theta}$ is larger than the ratio of major to minor torus radius, $R / a$, and the most dangerous helical deformations of the plasma cannot form because of the periodicity conditions. At about the same time a second approach appeared: the Reversed Field Pinch (RFP) in which $B_{\phi} \cong B_{\Theta}$ but the toroidal field component in the outer region has the opposite sign to that on the magnetic axis, thus creating a high shear of the field lines and forming a stable radial distribution of the magnetic field.

Fig. 1 - Cylindrical geometry.

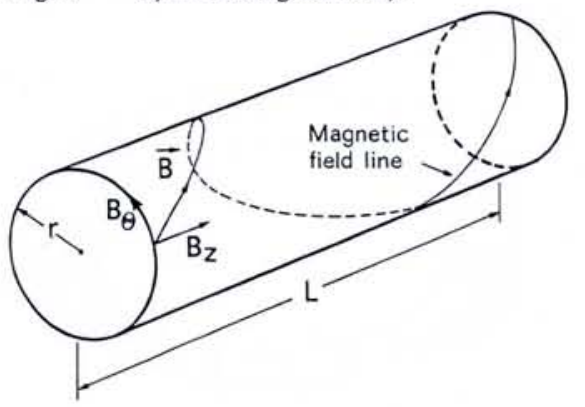

Table 1 - RFP devices in operation, under construction and contemplated

\begin{tabular}{|l|c|c|c|c|c|c|c|c|}
\hline & $\begin{array}{l}\text { ETA BETA II } \\
\text { (Padova, Italy) }\end{array}$ & $\begin{array}{c}\text { TPE-IR (M) } \\
\text { (Sakura-Mura, } \\
\text { Japan) }\end{array}$ & $\begin{array}{c}\text { ZT-40 (M) } \\
\text { (los Alamos, } \\
\text { USA) }\end{array}$ & $\begin{array}{c}\text { HBTX-IA } \\
\text { (Culham, UK) }\end{array}$ & $\begin{array}{c}\text { OHTE } \\
\text { (General Atomic, } \\
\text { USA) }\end{array}$ & $\begin{array}{c}\text { RFX } \\
\text { (Padova, Italy) }\end{array}$ & \multicolumn{2}{|l|}{ REACTOR } \\
\hline Torus Major Radius, m & 0.65 & 0.5 & 1.14 & 0.8 & 1.24 & 2 & 4.0 & 13 \\
Torus Minor Radius, m & 0.125 & 0.09 & 0.2 & 0.26 & 0.19 & 0.5 & 0.75 & 1.5 \\
Plasma Current, MA & 0.25 & 0.15 & 0.6 & 0.5 & 0.5 & 2 & 18.5 & 20 \\
First Operation & 1979 & 1980 & 1981 & 1981 & 1981 & 1988 & \\
\hline
\end{tabular}

The first large toroidal device in which RFP configurations were studied was ZETA (UK), which operated during the 1960s, where improved stability and confinement were observed when the plasma spontaneously generated a reversed toroidal field in the outer region. The importance of the reversed field configuration both experimentally and theoretically was better understood during the 1970s. A number of small toroidal devices were built and operated in that period (UK, USA, Italy, Japan) and many of the theoretical predictions of the MHD theory were experimentally confirmed.

During the same period, a major advance in our theoretical understanding of toroidal pinch discharges was made. It was realized that the most important property of the RFP is that the magnetic configuration is a minimum energy state to which, under certain conditions, the plasma can spontaneously relax. This basic property was first discussed in 1974 by J.B. Taylor who revived Woltjer's astrophysical theorem on force-free magnetic fields and extended it to laboratory plasma discharges. It is in practice this property of minimum energy state which gives to the RFP its favourable stability characteristics and makes it of particular interest among toroidal systems for magnetic confinement.

A new generation of experiments has become operational during the last several years (see Table 1) and it is now generally observed that the RFP configuration can confine relatively high $\beta$ plasmas, heated by ohmic heating only, with moderate magnetic fields, $B_{\phi} \cong B_{\Theta} \leq$ 1T. ( $\beta$ is the ratio between the plasma kinetic pressure and the magnetic field pressure. For a fusion reactor to be economic it should be greater than about $10 \%$.)

The temperatures and confinement times achieved ( $T \leq 0.6 \mathrm{keV}, \tau_{E} \leq 0.3$ $\mathrm{ms}$ ) are still modest compared to those required for thermonuclear fusion ( $T \cong$ $10 \mathrm{keV}, \tau_{E} \cong 1 \mathrm{~s}$ ) and to those achieved in present day tokamaks ( $T \cong 5 \mathrm{keV}, \tau_{E}$ $\cong 0.1 \mathrm{~s}$ ), but there is now a firmer scientific basis for extending RFP research to bigger systems and higher currents.

While the scientific feasibility of controlled thermonuclear fusion will probably be demonstrated in the tokamak configurations ${ }^{1}$ ), it is now recognized that alternative schemes such as Mirrors $\left.{ }^{2}\right)$, Stellarators ${ }^{3}$ ) and the RFP may offer more advantageous solutions for nuclear fusion reactors.

The RFP like the tokamak is a toroidal axisymmetric configuration but, whereas in the tokamak the plasma current is limited by stability requirements:

$$
I<2 \pi a^{2} B_{\phi} / R \mu_{o}
$$

the RFP can use high currents and powerful ohmic heating to produce relatively high- $\beta$ ( 10\%) plasmas without the need for either very high toroidal fields or powerful auxiliary heating methods. Furthermore it is now clear that although the RFP originally was thought to be intrinsically a pulsed device, the same processes which can lead to the formation of the configuration can also maintain it and give quasi steady state operation.

\section{Equilibrium}

In the RFP the distributions of both the poloidal and toroidal field components

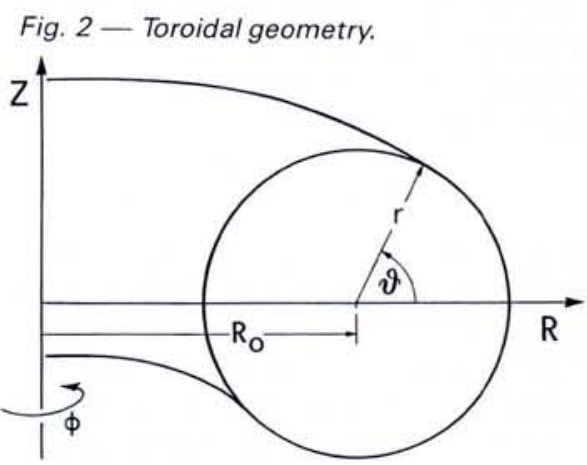


are mostly determined by currents flowing in the plasma, the total current density being almost parallel to the magnetic field. For an ideal equilibrium in toroidal geometry (Fig. 2), the magnetic surfaces are nested toroidal surfaces (Fig. 3 ) on which the magnetic field lines are helically wound with a pitch length (for a circular cross section of the magnetic surfaces):

$$
P=r B_{\phi} / B_{\Theta}=q R=\Delta \phi R / 2 \pi
$$

where $\triangle \phi$ is the change in toroidal angle of a magnetic line after traversing one poloidal circuit and $q$ represents the number of times the field line encircles the major axis in making one turn of the minor axis. It is worth noting that in a tokamak, $q \geq 1$ (typically $\sim R / a$ ) and in a RFP, $q<1$ (typically $\leq a / R$ ). Moreover in the RFP, the helical field lines have different pitches on the various magnetic surfaces (i.e. $q$ is a function of minor radius) and the direction in the outer region is opposite to that in the central part of the toroidal plasma; this is illustrated in Fig. 4.

RFPs can have a large aspect ratio $R / a$ and therefore the radial pressure balance in the poloidal plane can be described in the simpler geometry of a straight cylinder (Fig. 1). In this case the equilibrium equation for the poloidal beta which is defined as:

$$
\beta_{\Theta}=\frac{2 \mu_{0} \int_{0}^{a} p 2 \pi r d r}{\pi a^{2} B_{\Theta}^{2}(a)}
$$

where $p$ is the plasma pressure is:

$$
\beta_{\Theta}=1+\frac{1}{\Theta^{2}}\left[F^{2}-\frac{\left\langle B_{z}{ }^{2}\right\rangle}{\left\langle B_{z}\right\rangle^{2}}\right]
$$

The two parameters $F$ and $\Theta$ which characterize the pinch are defined as: $\left.F=B_{z}(a)<B_{z}\right\rangle$ and $\left.\Theta=B_{\Theta}(a) /<B_{z}\right\rangle$. These equations reduce with $\beta_{\phi}=1$ to the Bennett equilibrium equation:

$$
I^{2}=\frac{4 \pi}{\mu_{0}} 2 N k\left(T_{\mathrm{e}}+T_{\mathrm{i}}\right)
$$

where $N$ is the total number of particles per unit length and $\left(T_{e}+T_{i}\right)$ is averaged over all these particles.

In the case of the tokamak with $B_{z}$ almost uniform and so $F^{2} \cong 1$, equilibria with $\beta_{\Theta} \cong 1$ are possible, whereas in the RFP, characterized by $F^{2} \cong 0$ and $\left\langle B_{z}^{2}\right\rangle\left|\left\langle B_{z}\right\rangle^{2}\right\rangle 1$, the pressure balance condition requires $\Theta>1$ and in general $\beta_{\Theta}<\left(1-1 / \Theta^{2}\right)$. The total $\beta$ is related to the poloidal beta by:

$$
\beta_{\Theta} \cong\left[1+\left(\frac{q R}{a}\right)^{2}\right] \beta
$$

so that in the tokamak $\beta<<\beta_{\Theta}$, whereas in the RFP they are equal within a factor of two.

The problem of toroidal equilibrium consists of compensating the outward force arising from the gas kinetic expan- sion and the hoop force of the toroidal current. Equilibrium can be established through the influence of a conducting wall or a vertical field. If the plasma is surrounded by a perfectly conducting shell, the equilibrium is achieved when the plasma shifts far enough that the increased magnetic pressure, due to the compression of poloidal flux, balances the outward force. In the RFP, because $\beta_{\Theta}$ is small and $a / R$ can be small, only a small outward shift (typically a few percent of the plasma minor radius) is necessary to compress sufficient flux for toroidal equilibrium.

\section{Stability}

Obviously a plasma confined by a magnetic field away from the cold first wall cannot be in the lowest energy state as that would be characterized by thermal equilibrium. Confinement studies aim then to find dynamical equilibria with inhomogeneous plasma, and magnetic field spatial distributions which can be stable for times longer than the energy and particle confinement time.

The spatial gradients of the plasma kinetic pressure and the current density distribution are the two main sources of macroscopic instabilities.

The stability for localised pressure driven modes can be discussed on the basis of Suydam's criterion. This states that in cylindrical geometry, stability is obtained when

$$
\frac{1}{2}\left(\frac{r d q / d r}{\mathrm{q}}\right)^{2}>-\frac{r d p / d r}{B_{z}^{2} / 2 \mu_{0}}
$$

and shows that the destabilizing effect of the negative pressure gradient can be compensated by a sufficiently large shear $(d q / d r)$ in the magnetic field.

It is also evident that if $d q / d r=0$ when $d p / d r<0$, the Suydam criterion cannot be satisfied. This can be avoided when there is a vacuum region separating the plasma from the conducting wall if there is a reversal of $B_{z}$. The reason is that in vacuum, $q=q(a)(r / a)^{2}$, where $a$ is the radius of the plasma, whereas in the central region of the RFP, $q$ decreases with $r$, implying a minimum in the radial profile unless $q$ changes sign in the outer region. This is achieved by the reversal of the toroidal field which effectively maintains the shear high in the outer portion of the configuration.

A detailed study of the MHD stability for pressure driven and current driven modes shows that RFP profiles completely stable to both are possible with average total beta as high as $20 \%$. However it should be noted that if the finite resistivity of the plasma is taken into account, instability sets in at pressure gra-

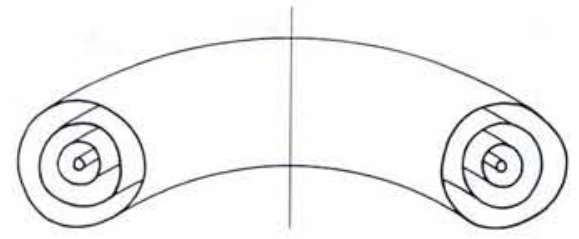

Fig. 3 - Toroidal magnetic flux surfaces.

dients substantially smaller than the critical value set by the Suydam's criterion.

\section{Minimum Energy State}

Although stable high $\beta$ equilibrium profiles exist theoretically, they are difficult to achieve experimentally by direct programming of the toroidal and poloidal electrical circuits. The main reasons are technological: for a full control of the profiles, programming on a short (MHD) timescale implying high voltages, would be required. However these RFP configurations have been demonstrated by J.B. Taylor to be minimum energy states to which, under certain conditions, the plasma can spontaneously relax.

For a plasma surrounded by a perfectly conducting wall, the minimum energy magnetic configuration is described by the solutions of the equation:

$$
\nabla \times \mathbf{B}=\mu \mathbf{B}
$$

with $\mu$ constant throughout the plasma. This configuration corresponds however to a large parallel current density flowing out to the wall. Also the $z$-component of the current density should substantially reverse in the outer region.

Experimentally, the magnetic field distribution in the central region of the plasma is of the form given by Taylor's theory, but not in the outer region where there is little or no current because the plasma temperature near the wall is usually low. Fig. 5 presents profiles typifying experimental findings for $\mu$ varying as $\mu=\left(2 \Theta_{0} / a\right)\left\{1-(r / a)^{4}\right\}$ with $\theta_{0}=$ 1.6. For this radial profile, the unrealistic features of the BFM are removed and one arrives at the conclusion that the experimental distributions are near minimum energy states.

Fig. 4-Magnetic field lines on an inner and an outer magnetic surface of a RFP configuration.

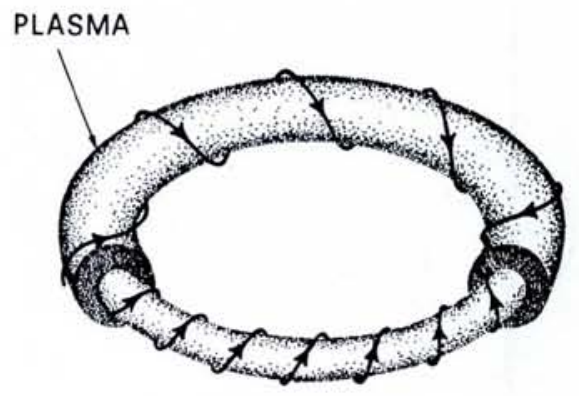


It is very important to notice that the relaxation to this near minimum energy distribution is a continuous process by which the field configuration can be maintained for times longer than the characteristic resistive diffusion time. In fact in a stable plasma described by classical MHD transport, a steady-state RFP could not be maintained. MHD turbulence or instabilities are required to drive the plasma currents which generate the field distribution. By analogy to the astrophysical and geophysical case, this is sometimes referred to as the dynamo process.

\section{Present and Future Experiments}

Recently, there has been much new information from five intermediate sized RFP machines $(a=9-26 \mathrm{~cm})$ at currents of a few hundred kA. In Table 1 are listed some of the main parameters of the various experiments along with the parameters of the RFX experiment and of two possible reactor units. In present experiments, the plasma parameters are: $T_{\mathrm{e}}$ is of the order of a few hundred $\mathrm{eV}$ (maximum $0.6 \mathrm{keV}$ ), $n_{\mathrm{e}}$ of the order of $10^{13}-10^{14} \mathrm{~cm}^{-3}, \beta_{\Theta} \cong 0.1$, with typical pulse lengths of $\sim 10 \mathrm{~ms}$, during which time the magnetic field configuration is sustained by the "dynamo" mechanism. The fluctuation level $(\delta B / B \cong 1 \%)$ is relatively low. The energy confinement times lie in the range $0.03-0.3 \mathrm{~ms}$.

One of the most important observations is the dependence of the plasma parameters on density. There exists a high filling density limit which may be compared to that in tokamaks and occurs at about the same value of the ratio of plasma current to plasma line density, $I / N \cong 10^{-14} \mathrm{~A} \mathrm{~m}$, beyond which the discharge is cold, resistive and radiation cooled because low- $Z$ impurities are not burnt through. In general, after an initial rise due to ionization, the density falls during the pulse, sometimes reaching an approximately constant value; in many experiments a large fraction of the initial density is lost. Essentially, although gas puffing (which is difficult in these experiments) and toroidal field control influences the density to some extent, it cannot be controlled externally.

The highest temperatures have so far usually been reached at low density; to reach high temperatures ( $\sim 1 \mathrm{keV}$ ) at high densities $\left(\approx 10^{14} \mathrm{~cm}^{-3}\right)$ currents in the MA range are required.

RFP experiments obtain typically values of $\beta_{\Theta} \cong 0.1$ fairly independently of the current in a range from 50 to $\sim 500$ kA. This corresponds approximately to a linear increase of the temperature with current and a scaling of the energy confinement time of the form $\tau_{E} \propto a^{2} T_{e}^{3 / 2}$.
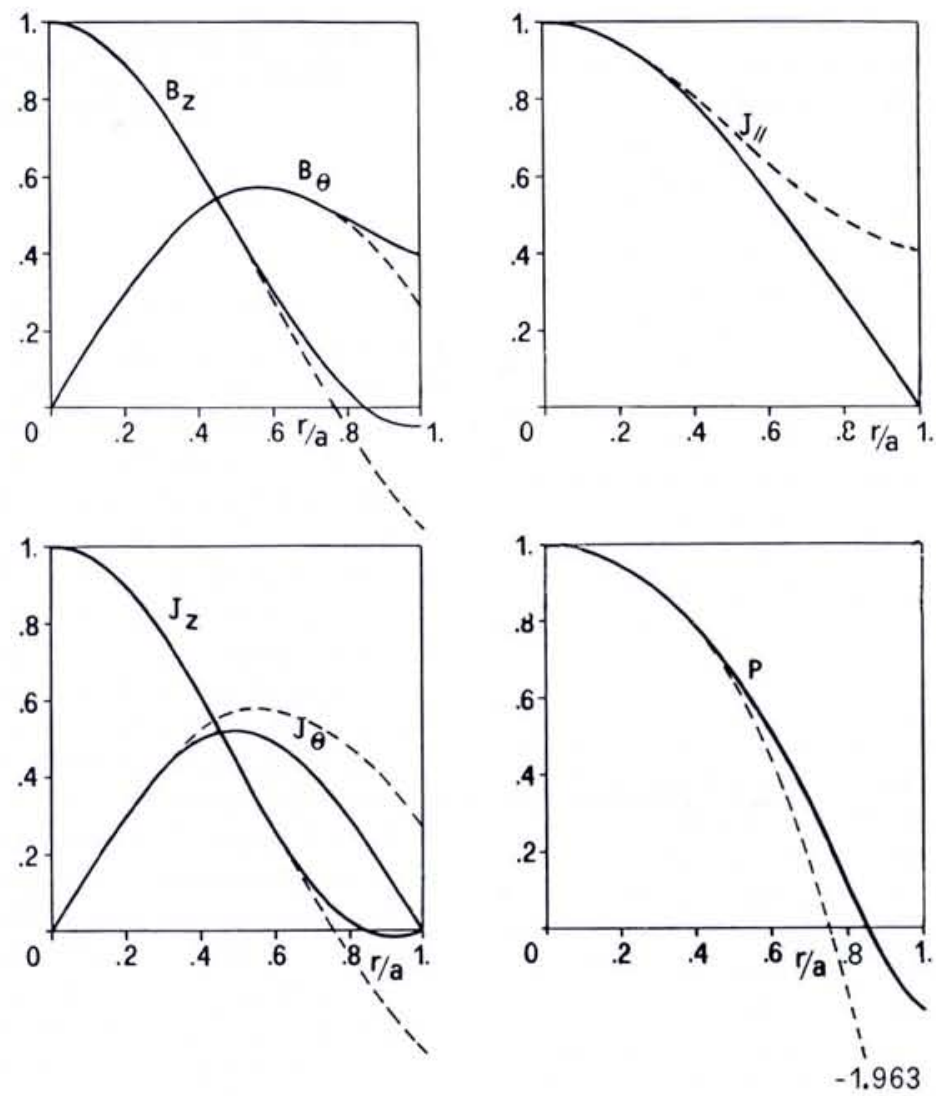

Fig. 5 - Comparison between typical RFP profiles (solid lines) and the theoretical BFM profiles (dashed lines).

Such a dependence is similar to that of the magnetic field diffusion time and to that of the classical ion heat conduction across the magnetic field due to Coulomb collisions.

This $\beta_{\Theta}=$ constant scaling of the energy confinement time is also consistent with the concept that the basic relaxation process, which maintains the RFP configuration near to the minimum energy state, occurs through resistive unstable modes whose characteristic time scale is the resistive diffusion time $\tau_{R} \propto a^{2} T_{e}^{3 / 2}$.

A comparison of ohmically heated tokamaks and pinches shows that small low current machines yield substantially higher values of temperature and energy confinement time in the tokamak mode, but with a much lower value of $\beta$. Extrapolating to larger experiments, with currents in the MA range, gives temperatures and energy confinement times which, with $\beta_{\Theta} \cong 0.1$, will be similar in the RFP and in ohmically heated tokamaks. However in the pinch these should be obtained with lower toroidal fields and larger values of total $\beta$ than would be obtainable in the tokamak by ohmic heating.

In general, low $\beta$ tokamaks provide good plasma confinement, but when their $\beta$ becomes comparable to that of an ohmically heated RFP the confine- ment deteriorates. If the RFP will maintain the presently achieved values of $\beta$ in a plasma with parameters closer to those of thermonuclear fusion, it will constitute a major alternative for a practical fusion reactor.

Hence the RFX experiment which received construction approval last October. It is a large RFP with a toroidal vessel of major and minor radii of $2 \mathrm{~m}$ and $0.5 \mathrm{~m}$ respectively, designed to operate with $2 \mathrm{MA}$ toroidal plasma current. It is being built at Padova and has been approved by the European Communities for priority action within the European fusion programme qualifying thereby for preferential support. It represents a substantial step in RFP research. Its objective is to demonstrate the possibility of producing temperatures and confinement times much closer to those required in a reactor and to compare the results with those from present day large tokamaks. The expected plasma parameters in RFX are $T \leq 1 \mathrm{keV}, n \cong$ $10^{14} \mathrm{~cm}^{-3}, \tau_{E} \cong 10 \mathrm{~ms}, \beta_{\Theta} \cong 10 \%$.

\section{In Conclusion}

The RFP's high beta capability, when combined with favourable topological features and the possibility for ohmic heating to ignition, may offer valuable options in fusion reactor development as they can lead to a less complex, less 
expensive, reactor scheme than the tokamak or stellarator with the possiblity of a smaller sized unit.

At the values of $\beta$ which are in principle possible in the RFP, the magnetic field will be relatively small and since the $B_{\Theta}$ field decreases outward with plasma radius as $1 / r$ the magnetic forces on external conductors can be quite modest. Since the aspect ratio is not restricted by stability considerations, an open structure with $R / a \cong 5-10$ can be used; this further eases the problems of magnetic forces and is advantageous for a reactor. If ignition by ohmic heating alone is possible, the complexity and cost of additional heating will be avoided, which is a major simplification.

Although as presently envisaged the RFP reactor would be pulsed, it is now clear that the magnetic field distribution is self-generating and this can lead to a quasi steady state operation. For full steady state operation, means must be found, as for all systems using transformer coupling, to drive the toroidal current continuously. Methods proposed for the tokamak using neutral beam or high frequency driven currents in principle might apply in a pinch, and will be investigated and tested at a more advanced stage of pinch development.

\section{REFERENCES}

1. Gibson A., Europhysics News 14 (1983) 4. 2. Baldwin D.E., Europhysics News 12 (1981) $8 / 9$.

3. Wobig H., Europhysics News 13 (1982) $8 / 9$.

\section{FURTHER READING}

- for early pinch work see Glasstone S., Lovberg R.H., Controlled Thermonuclear Reactions (D. Van Nostrand, Princeton, N.J.) 1960;

— for a summary of the results up to 1978 , see Ortolani S., Reversed Field Pinch Configuration, Nuclear Fusion 19 (1978) 535;

- for a general review of the RFP research up to 1979, see Bodin H.A.B. and Newton A.A., Reversed Field Pinch Research, Nuclear Fusion 20 (1980) 1255;

— for more updated reviews, see Bodin H.A.B. and Ortolani S., the Status of RFP Experiments, University of Padova Report UPee 82/09 (1982);

Dreicer H., Topics in Reversed FieldPinch Physics, Physica Scripta T2/2 (1982) 435; - for reports on progress in the major experiments, see the proceedings of the biennal IAEA Conferences on Plasma Physics and Controlled Nuclear Fusion Research;

- for a recent collection of papers on RFP theory and experiments see the proceedings of the Course and Workshop on Mirror Based and Field Reversed Approaches to Magnetic Fusion, Varenna, Sept. 7-17 (1983), to be published by the Commission of the European Communities.

\section{The Seventh European Regional Astronomy Meeting}

The Seventh European Regional Astronomy Meeting, organized by the European Physical Society and the International Astronomical Union, took place in Florence from December 12 - 16, 1983. It was cosponsored by the Italian Ministry of Education, Arcetri Astrophysical Observatory, the University of Florence, and the Italian Astronomical Society. The opening ceremony was quite spectacular since it was held in the old palace "Palazzo della Signoria", with trumpet players in Renaissance costumes behind the official table.

The EPS and IAU are interested in promoting scientific exchange between astronomers, astrophysicists and physicists, the borders between their disciplines often being difficult to define. One of the means of achieving this goal is to organize regular meetings on the European level: these meetings should also provide the young scientists with an opportunity to meet the more established, and to publicize their own work.

The IAU has been organizing European Regional Meetings for several years now;

- 1972, Athens: "Solar Activity and Related Interplanetary and Terrestrial Phenomena" - "Stars and the Milky Way System" _ "Galaxies and Relativistic Astrophysics".

— 1974, Trieste: "Stars and Stellar Evolution",

- 1975, Tbilisi: "Stars and Galaxies from Observational Points of View".

- 1978, Uppsala: "Stars and Star Systems".

— 1980, Liège: "Variability in Stars and Galaxies".

- 1982, Dubrovnik: "Sun and Planetary System".

The first six meetings were topical i.e. essentially devoted to one (sometimes very broad) subject whereas the seventh showed the result of a tighter collaboration between the EPS and the IAU, in the following sense: talks in any astrophysical discipline were accepted, and parallel sessions as well as invited talks were organized on topics at the frontier between astronomy and physics and/or in areas where new developments in physics are important for astrophysical research.

It is fair to say that this Seventh Regional Astronomy Meeting was a great success since it attracted over
400 participants, with the presentation of about 240 contributions. The invited papers will be published by the Societa Astronomica Italiana (Largo E. Fermi, 5, I-50125 Firenze).

During the last two days of the meeting, three full-day specialized sessions were organized on the following subjects:

- the NL-UK-USA Infrared Astronomy Satellite, IRAS;

- the European X-ray Satellite,

EXOSAT;

- Atomic and Molecular Physics for Astronomy.

\section{IRAS}

IRAS was launched on 25 January, 1983 , and ended its astronomical life on 22 November, 1983 when its coolant ran out, but in the course of the first six months, the spacecraft was able to view the entire celestial sphere. The cooled 22.4 in telescope mapped the area in bands centred around 12, 25, 60 and $100 \mu \mathrm{m}$ and made more detailed observations of several targets. There has been very little release of scientific data so far: first accounts are to appear in a series of papers in Astrophysical Journal Letters (March 1984), and a catalogue is scheduled for August-September 1984 (with positions and other information on more than 200000 far-infrared sources).

The specialized session organized in Florence gave the participants the occasion to listen to a series of talks which included several on comets and fast moving objects, the "minor planet" or burned out comet 1983 TB, a solar system dust ring, the "infrared cirrus" clouds (very cold dust at $\cong 35^{\circ} \mathrm{K}$, present even in the direction of the galactic poles), galactic cold dust (part of the "missing mass"?), stars with dust shells (ring of solid particles around Vega, older clouds at larger distances around Betelgeuse), the Crab Nebula, sources in Molecular Clouds, the Andromeda Galaxy M31, infrared galaxies (a huge number of them visible at the long wave bands), clusters and quasars, and the intriguing blank fields (IR sources with presently no optical counterpart). After these presentations of IRAS results, it was very clear that the astronomical community was anxiously waiting for the release of the first papers and of the catalogue since 